\title{
Saturação de Oxigênio Fetal Medida pela Oximetria de Pulso durante 0 Trabalho de Parto: Relações com o pH da Artéria Umbilical
}

\author{
Fetal Oxygen Saturation Measured by Pulse Oximetry during Labor: Relation to Umbilical Artery \\ $\mathrm{pH}$
}

Edson Nunes de Morais,Francisco Maximiliano Pancich Gallarreta, Patricia Spara

\begin{abstract}
RESUMO
Objetivos: estudar os niveis de saturação de oxigênio fetal $\left(\mathrm{SpO}_{2}\right)$ durante o trabalho de parto pela técnica da oximetria de pulso e sua relação com o $\mathrm{pH}$ da artéria umbilical (AU). Pacientes e Métodos: $a \mathrm{SpO}_{2}$ fetal foi medida durante o parto por meio da técnica da oximetria de pulso em 50 casos. Comparou-se a média dos valores de $\mathrm{SpO}_{2}$ entre os dois periodos do trabalho de parto, sendo o primeiro subdividido em fases, segundo a dilatação cervical $\leqslant 4$ $\mathrm{cm}, 5-7 \mathrm{~cm}$ e 8-9 cm). Os valores de $\mathrm{SpO}_{2}$ foram estudados em função do $\mathrm{pH}$ da $\mathrm{AU}$ ao nascimento $(\geq 7,20$ e $<7,20)$. Considerou-se como normal uma $\mathrm{SpO}_{2} \geq 30,0 \%$.

Resultados: as médias da $\mathrm{SpO}_{2}$ fetal no primeiro periodo do parto foram de $53,0 \pm 7,3 \%$ e 44,2 \pm 6,8\%, e no segundo 46,8 \pm 7,7\% e 38,4 \pm 7,1\% (pH da $A U \geq 7,20$ e <7,20, respectivamente; $p<0,01)$. Quando o primeiro periodo foi subdividido, as médias de $\mathrm{SpO}_{2}(\mathrm{pH}$ de $A U \geq 7,20)$ foram de 55, $1 \pm 5,1 \%(\leq 4 \mathrm{~cm}), 52,3 \pm 4,6 \%(5-7 \mathrm{~cm})$ e 51,5 \pm 7,2\% (8-9 cm); para um $\mathrm{pH}$ de $\mathrm{AU}<7,20$ as médias de $\mathrm{SpO}_{2}$ de 46,3 \pm 5,1\% ( $\left.\leq 4 \mathrm{~cm}\right), 43,6 \pm 6,7 \%(5-7 \mathrm{~cm})$ e 42,8 $\pm 5,8 \%(8-9 \mathrm{~cm})$. Considerando o $\mathrm{pH}$ da AU estas diferenças foram significantes $(p<0,01)$. Conclusões: houve um decréscimo significante dos valores de $\mathrm{SpO}_{2}$ fetal durante o trabalho de parto quando utilizada a técnica da oximetria de pulso.
\end{abstract}

PALAVRAS-CHAVE: Oximetria fetal de pulso. Monitorização fetal. Anoxia perinatal. Trabalho de parto.

\section{Introdução}

A avaliação clínica do concepto durante o trabalho de parto é usualmente realizada por meio

\footnotetext{
Departamento de Ginecologia e Obstetrícia do Centro de Ciências da Saúde da Universidade Federal de Santa Maria. Correspondência: Edson Nunes de Morais Av. Borges de Medeiros, 1699 / 604

97015-090 - Santa Maria - RS

Fone: (055) 222-1942 - Fax: (055) 220-8018

e-mail: edson@ccs.ufsm.br
}

da monitorização contínua ou de ausculta intermitente da freqüência cardíaca fetal (FCF). Quando a FCF encontra-se alterada, ou de dificil leitura no traçado de registro, outros métodos são utilizados para avaliar o feto, tais como o de Saling, para a análise bioquímica do sangue capilar do couro cabeludo, o da estimulação do couro cabeludo fetal, ou ainda o método do estímulo acústico ${ }^{4,5}$.

Várias tentativas foram realizadas no sentido de monitorizar continuamente o estado ácido-base fetal. As técnicas utilizadas experimentalmente mostraram-se limitadas, 
como no caso da monitorização do $\mathrm{pH}$ e da $\mathrm{pO}_{2}$ fetal, e não constituíram ferramentas clinicamente aplicáveis na prática obstétrica diária ${ }^{1,20,22}$.

A oximetria fetal de pulso é uma nova tecnologia atualmente em fase de desenvolvimento para a avaliação fetal intraparto ${ }^{7,11,12,15,19}$. Têm sido realizados estudos buscando o estabelecimento de valores de normalidade da saturação de oxigênio fetal durante o parto ${ }^{10,13}$. A oximetria fetal de pulso é um método não-invasivo para o feto, fornece resultados continuamente durante o trabalho de parto e pode melhorar a especificidade diagnóstica das condições fetais ao nascimento se associada à cardiotocografia.

O objetivo do presente trabalho foi o de estudar os niveis de saturação de oxigênio fetal durante o trabalho de parto pela técnica da oximetria de pulso, em relação ao $\mathrm{pH}$ da artéria umbilical.

\section{Pacientes e Métodos}

Efetuamos um estudo prospectivo no qual foram incluídas 50 pacientes em trabalho de parto atendidas no Centro Obstétrico do Serviço de Obstetrícia do Hospital Universitário de Santa Maria. Os critérios de inclusão das parturientes no protocolo de pesquisa foram: ser portadora de gestação única com idade gestacional $\geq 37$ semanas, apresentação de vértice, membranas amnióticas rotas espontânea ou artificialmente com menos de 12 horas do início da monitorização materno-fetal, dilatação cervical de pelo menos 2 $\mathrm{cm}$, altura da apresentação em pelo menos -1 de De Lee, e assinatura do termo de consentimento esclarecido.

Os critérios de exclusão foram: gestação com idade $<37$ semanas, presença de infecção genital clinicamente suspeitada por ocasião da internação; temperatura superior a $38^{\circ} \mathrm{C}$, placenta prévia, gemelaridade, sangramento vaginal de origem desconhecida, apresentação pélvica, situação transversa, mais de 1 cesárea prévia, dilatação cervical $<2 \mathrm{~cm}$, altura da apresentação acima de -1 de De Lee e as não-signatárias do termo de consentimento esclarecido.

$O$ registro da atividade uterina era obtido mediante colocação de tocotransdutor no abdômen materno, na altura do corno uterino oposto ao lado do dorso fetal. O registro em papel termo-sensivel era iniciado após a calibração empírica do cardiotocógrafo em $10 \mathrm{mmHg}$. Monitorizaram-se 7.970 minutos, ou 132,8 horas, com uma média de 159,4 $\pm 107,5$ minutos, ou 2,6 $\pm 1,6$ horas. A freqüência cardiaca fetal foi monitorizada pelos métodos externo e/ou interno. A cardiotocografia externa era realizada pela colocação de transdutor (ultra-som) de FCF no abdômen materno, na altura do dorso fetal. A cardiotocografia interna, quando indicada em casos de alteração da FCF detectada pelo método externo ou por registro externo de qualidade insatisfatória, era realizada pela colocação de eletrodo em espiral de Hon tipo 1, no couro cabeludo fetal, na altura de um dos parietais, cuidando para que a inserção ocorresse longe das fontanelas. Como critérios de interpretabilidade dos traçados cardiotocográficos, utilizaram-se aqueles recomendados pelo "National Institute of Child Health and Human Development Research Planning Workshop" (NICHD) 9 .

A saturação de oxigênio fetal $\left(\mathrm{SpO}_{2}\right)$ foi registrada por método da oximetria fetal de pulso. Rotas as membranas amnióticas, espontânea ou artificialmente, era feito toque obstétrico com a finalidade diagnóstica de variedade de posição fetal e altura da apresentação. Em seguida iniciava-se o procedimento de colocação intra-uterina de oxisensor de pulso. O corpo do sensor, entre os dedos indicador e médio do obstetra, era introduzido lenta e delicadamente através do colo uterino, entre a apresentação e a parede uterina. A introdução do equipamento era realizada até que a saliência tátil no cabo do sensor se colocasse na entrada do canal cervical, o que denotava o posicionamento do corpo do sensor na altura da face fetal. Após obtida uma boa qualidade de sinal de saturação de oxigênio fetal, iniciava-se o registro oximétrico, que deveria se desenvolver por todo o restante do trabalho de parto. Um oxisensor era utilizado somente em uma única paciente.

Considerou-se como saturação de oxigênio dentro dos limites da normalidade, quando o traçado oximétrico apresentava valores de saturação de $\mathrm{O}_{2} \geq 30 \%$. Como alteração da saturação de oxigênio foram considerados niveis inferiores a $30 \%$ entre as contrações uterinas, por tempo superior a 10 minutos consecutivos ${ }^{8}$. Para se interromper a gravidez por sofrimento fetal agudo, levou-se em consideração tão somente a alteração da saturação de oxigênio fetal ou por alterações patológicas da FCF quando perdida a qualidade do sinal de registro da $\mathrm{SpO}_{2}$. No presente estudo a amostra era constituída apenas de casos com $\mathrm{SpO}_{2} \geq 30 \%$.

Para a análise dos valores da saturação de oxigênio conceptual durante as várias fases do trabalho de parto procedia-se da seguinte forma: no papel de registro oxicardiotocográfico, encontravam-se assinalados a fase do trabalho de parto (dada em $\mathrm{cm}$ de dilatação cervical pelo observador) e o registro gráfico da $\mathrm{SpO}_{2}$ fetal. Em 
cada fase $(\leq 4 \mathrm{~cm}, 5-7 \mathrm{~cm}, 8-9 \mathrm{~cm}$ e $10 \mathrm{~cm})$, consignava-se o valor médio da $\mathrm{SpO}_{2}$, dado pelo somatório dos pontos registrados a cada 30 segundos, e dividia-se pelo número de pontos. Os casos foram analisados levando-se em conta que o último registro de $\mathrm{SpO}_{2}$ deveria ocorrer no máximo até 30 minutos do desprendimento fetal e com pelo menos 20 pontos identificados.

O sangue de artéria e veia umbilical era obtido através do clampeamento do cordão (duas pinças próximas ao coto umbilical e uma próxima ao intróito vaginal) antes que o recém-nascido fizesse o seu primeiro movimento inspiratório. Seccionado o cordão, coletava-se sangue de artéria e veia umbilical em seringas previamente heparinizadas, em quantidade suficiente para a medida de $\mathrm{pH}$ e demais componentes do equilibrio ácido-base. Considerou-se como padrão bioquímico alterado ao nascimento um $\mathrm{pH}$ de artéria umbilical $<7,20^{3}$.

Para o registro da atividade uterina, freqüência cardíaca fetal e saturação de oxigênio fetal utilizou-se um cardiotocógrafo da Corometrics Medical Systems, Inc., modelo 129-F. A $\mathrm{SpO}_{2}$ era obtida pela utilização de um oxi-sensor de pulso da Nellcor Puritan Bennett, Inc., Pleasanton, CA, USA, modelo FS-14B. Para a medida dos parâmetros do equilíbrio ácido-base do sangue dos vasos umbilicais, utilizaram-se aparelhos AVL, Automatic Blood Gas System, modelos 990 e Compact 1, do Laboratório Central de Análises Clínicas do Hospital Universitário de Santa Maria.

A presente pesquisa foi devidamente aprovada pelas Comissões de Pesquisa e de Ética do Centro de Ciências da Saúde da Universidade Federal de Santa Maria.

Os resultados foram analisados por estatística descritiva, para dados de interesse geral, e pelo teste $t$ de Student para amostras independentes. $O$ nivel de significância consignado foi para um $\mathrm{p}$ de $5 \%$.

\section{Resultados}

Nos 50 casos a média de $\mathrm{SpO}_{2}$ fetal nas várias fases do trabalho de parto esteve acima de $30 \%$. Todos os recém-nascidos apresentaram Apgar de $1^{\circ}$ e $5^{\circ}$ minuto $\geq 7$, com exceção de 1 recém-nascido que apresentou no primeiro minuto Apgar 5 .

Quanto à idade, metade das pacientes encontrava-se na faixa inferior aos 25 anos. A média de idade das parturientes foi de 24,7 $\pm 7,1$ anos. Em mais de $50 \%$ dos casos a idade gestacional era $\geq 40$ semanas. A média da idade gestacional foi de 39,5 $\pm 1,4$ semanas.

A maior parte da amostra era de gestantes nuliparas $(46,0 \%)$ e primíparas $(24,0 \%)$. A paridade variou entre 1 e 8 nascimentos. As faixas de freqüência do peso fetal ao nascimento encontramse representadas na Figura 1. A grande maioria dos recém-nascidos $(60 \%)$ apresentou peso entre 3.000 e 3.999 gramas. A média de peso dos recém-nascidos foi de 3.288, com limites entre 2.510 e 4.470 gramas.

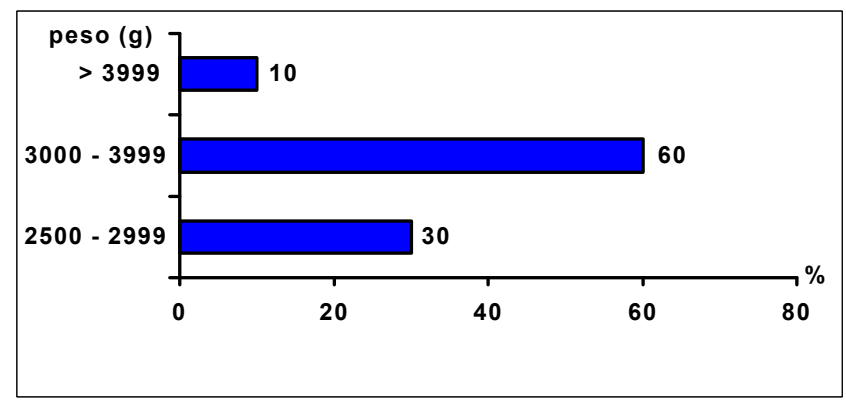

Figura 1 - Distribuição de 50 parturientes submetidas à monitorização pela oximetria fetal de pulso, segundo o peso fetal.

Das 50 grávidas estudadas, $62 \%$ tiveram seus partos de forma espontânea por via vaginal; em $38 \%$ das vezes o parto foi operatório $(24 \%$ de fórcipe e $14 \%$ de cesárea) conforme demonstra a Figura 2. As indicações de cesárea foram a desproporção céfalo-pélvica, 42,8\% (3 casos); parada de progressão do trabalho de parto, $28,6 \%$ ( 2 casos), distocia de rotação, 28,6\% (2 casos).

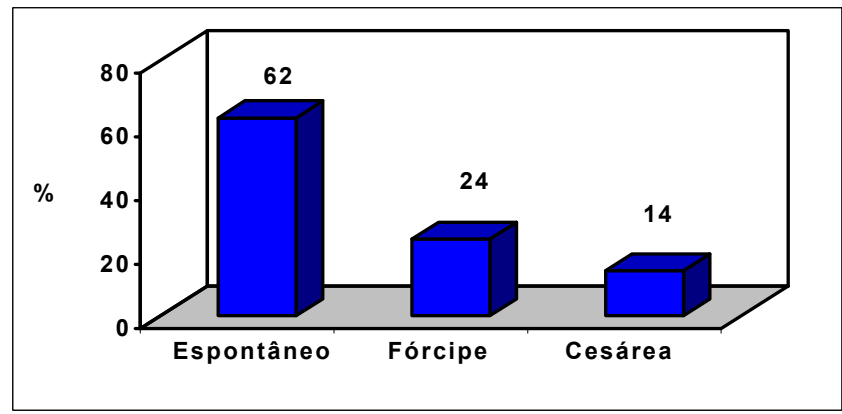

Figura 2 - Distribuição de 50 parturientes submetidas à monitorização pela oximetria fetal de pulso, segundo o tipo de parto.

Na Tabela 1 encontra-se a distribuição dos valores médios e desvios padrão dos parâmetros de gasometria do sangue da artéria umbilical de 50 recém-nascidos que foram monitorizados pela oximetria fetal de pulso, cujos valores de $\mathrm{SpO}_{2}$ eram iguais ou superiores a 30\% durante o trabalho de parto, em função do $\mathrm{pH}$ da artéria umbilical $\geq 7,20$ ou $<7,20$. Observa-se que, embora a saturação de oxigênio fetal estivesse dentro dos limites da normalidade, uma porcentagem importante de recém-nascidos apresentaram ph da artéria umbilical inferior a 7,20 $(24,5 \%)$. 
Tabela 1 - Distribuição das médias \pm DP dos valores dos parâmetros do equilíbrio ácido-base do sangue da $\mathrm{AU}$ de 50 recém-nascidos com $\mathrm{SpO}_{2} \geq 30 \%$, durante 0 trabalho de parto, segundo o pH do sangue da AU.

\begin{tabular}{|c|c|c|c|c|c|c|}
\hline $\mathrm{pH} A U$ & $\mathrm{pH}$ & $\mathrm{pO}_{2}$ & $\mathrm{pCO}_{2}$ & $\mathrm{HCO}_{3}$ & DB & $\mathrm{SaO}_{2}$ \\
\hline$<7,20(\mathrm{n}=12)$ & $7,11( \pm 0,06)$ & $19,6( \pm 7,5)$ & $61,4( \pm 14,3)$ & $19,2( \pm 4,1)$ & $-9,8( \pm 4,1)$ & $16,2( \pm 13,5)$ \\
\hline$\geq 7,20(\mathrm{n}=38)$ & $7,27( \pm 0,05)$ & $19,1( \pm 5,8)$ & $46,6( \pm 10,8)$ & $21,1( \pm 5,1)$ & $-3,6( \pm 3,1)$ & $23,1( \pm 10,5)$ \\
\hline
\end{tabular}

DP: desvio padrão; $\mathrm{AU}$ : artéria umbilical; $\mathrm{pO}_{2}$ : pressão parcial de oxigênio; $\mathrm{pCO}_{2}$ : pressão parcial de gás carbônico; $\mathrm{HCO}_{3}$ : bicarbonato; $\mathrm{DB}$ : déficit de bases; SaO ${ }_{2}$ : saturação de oxigênio.

Os valores médios e os desvios padrão da $\mathrm{SpO}_{2}$ de 50 recém-nascidos monitorizados pela oximetria fetal de pulso encontram-se distribuídos na Figura 3 , segundo as várias fases do primeiro periodo e segundo período. Estes dados estão relacionados ao $\mathrm{pH}$ da $\mathrm{AU}$ ao nascimento $(\geq 7,20$ e $<7,20)$. Houve diferença significante entre os valores médios da saturação de oxigênio fetal entre o primeiro e o segundo período do parto, independentemente se o $\mathrm{pH}$ da $\mathrm{AU}$ era $\geq 7,20(53,0 \pm 7,3 \%$ e $46,8 \pm 7,7 \%$, respectivamente; $\mathrm{p}<0,05)$ ou $<7,20(44,2 \pm 6,8 \%$ e $38,4 \pm 7,1 \%$, respectivamente; $p<0,01)$. Da mesma forma foi significante a diferença dos valores entre os grupos estudados, segundo o $\mathrm{pH}$ da AU. Nas diferentes fases do primeiro período observou-se uma tendência ao decréscimo dos níveis de $\mathrm{SpO}_{2}$, não sendo, porém, significantes essas diferenças, tanto para o grupo com $\mathrm{pH}$ da $\mathrm{AU} \geq 7,20(\leq 4 \mathrm{~cm}=55,1 \pm$ $5,1 \% ; 5-7 \mathrm{~cm}=52,3 \pm 4,6 \%$; e $8-9 \mathrm{~cm}=51,5 \pm 7,2 \%)$ como para o grupo com $\mathrm{pH}$ da $\mathrm{AU}<7,20(\leq 4 \mathrm{~cm}=46,3$ $\pm 5,1 \% ; 5-7 \mathrm{~cm}=43,6 \pm 6,7 \% ; 8-9 \mathrm{~cm}=42,8 \pm 5,8 \%)$, conforme dados da Tabela 2 . Ainda que nos limites da normalidade, a saturação de oxigênio fetal se mostrou menor naqueles fetos cujo $\mathrm{pH}$ do sangue da artéria umbilical foi $<7,20$. Por outro lado, o Apgar destes recém-nascidos foi igual ou superior a 7 no primeiro e quinto minuto de vida, com exceção de 1 recém-nascido com Apgar 5 no $1^{\circ}$ minuto.

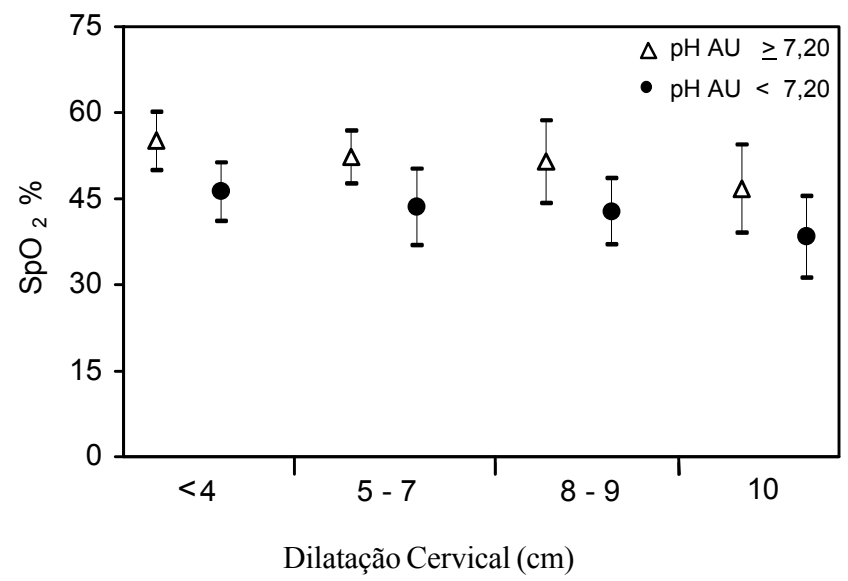

Figura 3 - Médias e desvios padrão da saturação de oxigênio fetal durante o parto segundo a dilatação cervical e o pH da artéria umbilical
Tabela 2 - Distribuição das médias e DP dos valores de $\mathrm{SpO}_{2}$ de 50 fetos, em função do $\mathrm{pH}$ da $\mathrm{AU}<7,20$ ou $\geq 7,20$, durante o primeiro ( $D C$ entre $\leq 4$ e $9 \mathrm{~cm}$ ) e segundo periodos $(D C$ de $\geq 10 \mathrm{~cm}$ ) do trabalho de parto.

\begin{tabular}{ccccc}
\hline \multicolumn{5}{c}{$\mathrm{SpO}_{2}(\%)$} \\
DC & $\leq 4$ & $5-7$ & $8-9$ & 10 \\
& $\mathbf{c m}$ & $\mathbf{c m}$ & $\mathbf{c m}$ & $\mathbf{c m}$ \\
\hline$<7,20(n=12)$ & $\begin{array}{c}46,3 \pm 5,1 \\
(n=5)\end{array}$ & $\begin{array}{c}43,6 \pm 6,7 \\
(n=9)\end{array}$ & $\begin{array}{c}42,8 \pm 5,8 \\
(n=12)\end{array}$ & $\begin{array}{c}38,4 \pm 7,1 \\
(n=10)\end{array}$ \\
$\geq 7,20(n=38)$ & $\begin{array}{c}55,1 \pm 5,1 \\
(n=25)\end{array}$ & $\begin{array}{c}52,3 \pm 4,6 \\
(n=34)\end{array}$ & $\begin{array}{c}51,5 \pm 7,2 \\
(n=38)\end{array}$ & $\begin{array}{c}46,8 \pm 7,7 \\
(n=35)\end{array}$ \\
Significância & $p<0,01$ & $p<0,01$ & $p<0,001$ & $p<0,001$
\end{tabular}

DP: desvio padrão; AU: artéria umbilical; DC: dilatação cervical.

Não procuramos relacionar os valores de $\mathrm{SpO}_{2}$ fetal e gasometria dos vasos umbilicais com os padrões de freqüência cardiaca fetal $(\mathrm{FCF})$. No entanto, é de se registrar que em 17 casos (34\%) a FCF encontrou-se alterada, segundo os critérios do $\mathrm{NICHD}^{15}$, ao passo que a $\mathrm{SpO}_{2}$ fetal média permaneceu normal.

\section{Discussão}

A monitorização eletrônica da FCF durante o trabalho de parto constitui ainda o método mais utilizado pelos obstetras para a avaliação do bemestar conceptual. Apesar da boa sensibilidade, são preocupantes as taxas de falso-positivos, fazendo com que a especificidade para a detecção de hipóxia e acidemia fetais seja relativamente baixa. O padrão mais ominoso da FCF associa-se no máximo com uma incidência de $50-65 \%$ de recém-nascidos deprimidos, definidos pelo índice de Apgar. Uma análise custo-benefício da FCF realizada por Banta e Thacker em $1979^{2}$ demonstrou um valor preditivo de $99,7 \%$ para um Apgar $>6 \mathrm{com}$ base numa FCF normal, e de $50 \%$ para um Apgar $<7$ com base numa FCF anormal.

Em nosso material observamos 17 casos com FCF alterada (segundo as normas recomendadas 
pelo "National Institute of Child Health and Human Development Research Planning Workshop") $)^{9}$ e que, em primeira instância, poderiam ser considerados como potencialmente cirúrgicos. Tal análise, no entanto, não foi objeto deste estudo.

A oximetria fetal de pulso, técnica de desenvolvimento recente, tem-se mostrado importante para minimizar as limitações da cardiotocografia. No entanto, os valores de normalidade da saturação de oxigênio fetal durante o trabalho de parto ainda estão por serem estabelecidos de forma conclusiva, uma vez que variam amplamente durante o trabalho de parto.

Estudos experimentais e em humanos têm sido desenvolvidos para o estabelecimento do nível crítico de $\mathrm{SpO}_{2}$ fetal. Oeseburg et al. ${ }^{17}$ e Nijland et al. ${ }^{16}$ realizaram uma série de pesquisas neste sentido em fetos de ovelha. Reduzindo o volume de oxigênio materno inspirado ou reduzindo o fluxo sangüineo uterino materno, por oclusão vascular, estes autores demonstraram que, sob uma saturação de $\mathrm{O}_{2}$ no sangue fetal igual ou superior a $30 \%$, o seu metabolismo aeróbico era mantido. O déficit de base aumentava numa razão de $-0,5$ nmol/1 por 10 minutos, quando a saturação de oxigênio sofria queda abaixo do limite de $30 \%$. Estes estudos foram confirmados por Richardson et al. ${ }^{18}$ Investigações em humanos desenvolvidas por vários autores corroboram os resultados encontrados em animais de experimentação. Niveis iguais ou superiores a $30 \%$ de saturação de oxigênio fetal intraparto, são atualmente considerados pelos autores como limite da normalidade.

Dildy et al. ${ }^{7}$ em estudo preliminar de 73 casos relatam niveis médios de $\mathrm{SpO}_{2}$ fetal durante o trabalho de parto de 57,9 $\pm 10,0 \%$. Langer et al. ${ }^{14}$, estudando 54 pacientes nos últimos 30 minutos do parto, observaram uma média de $\mathrm{SpO}_{2}$ de 41,2 \pm $10,0 \%$. Posteriormente, Dildy et al..$^{6,8}$ estudaram as fases do primeiro período e o segundo período do parto. Os valores médios de $\mathrm{SpO}_{2}$ no primeiro periodo foram de $59 \pm 10,0 \%$ e no segundo periodo de $53,0 \pm 10,0 \%$, para fetos com resultados perinatais considerados normais. Quando dividiram o primeiro período em fases observaram, para uma dilatação cervical igual ou inferior a $4 \mathrm{~cm}$, níveis de $62 \pm 9,0 \%$; de 5 a $7 \mathrm{~cm}$, níveis de $60 \pm 11 \%$; e entre 8 e $10 \mathrm{~cm}, 58 \pm 10 \%$. A média do $\mathrm{pH}$ da artéria umbilical neste grupo de estudo foi de 7,25 $\pm 0,05$.

No presente estudo observamos que os fetos com pH da AU abaixo de 7,20 (12 casos) apresentaram niveis de $\mathrm{SpO}_{2}$ inferiores aos fetos com $\mathrm{pH} \geq 7,20$, tanto no primeiro como no segundo periodo do parto, e esta diferença foi significante. A média do $\mathrm{pH}$ da AU nestes casos situou-se em $7,11 \pm 0,58$. Por outro lado observa-se que valores de $\mathrm{SpO}_{2}$ permaneceram dentro da normalidade, isto é, acima de $30 \%$ de saturação de oxigênio. Semelhante aos relatos de literatura, observamos uma $\mathrm{SpO}_{2}$ do primeiro período superior à encontrada no segundo período, independente dos valores de $\mathrm{pH}$ da AU.

Van den Berg et al. ${ }^{21}$, em um estudo multicêntrico envolvendo Holanda, Estados Unidos, Alemanha e Inglaterra, descrevem uma taxa de 57\% de partos vaginais espontâneos, de $29 \%$ de partos vaginais operatórios e de $14 \%$ de cesárea, para uma população de 308 gestantes. Em apenas 6 casos o sofrimento fetal foi a causa da operação cesariana.

Em nosso material, ainda que constituído de fetos sem diagnóstico de sofrimento pela leitura oximétrica, a cesárea foi realizada em 7 oportunidades, não sendo diferente da incidência dos autores citados. Por outro lado, o número de partos operatórios vaginais mostra-se elevado, o que pode ser explicado pelo fato de assistirmos por mais tempo um segundo período do parto com niveis de $\mathrm{SpO}_{2}$ dentro da normalidade, apesar de alterações importantes da FCF neste período eventualmente ocorrerem. Desta forma, muitos partos instrumentados foram indicados para reduzir o tempo do segundo período, levando também em consideração o componente materno de desgaste físico.

Concluindo, nossos resultados mostram um comportamento da $\mathrm{SpO}_{2}$ fetal durante o trabalho de parto com tendência à diminuição de seus valores à medida em que evolui o parto. Esta tendência se dá tanto em fetos com $\mathrm{pH}$ da $\mathrm{AU} \geq 7,20$ como naqueles com $\mathrm{pH}$ da $\mathrm{AU}<7,20$. Demonstram ainda que, para esses limites de $\mathrm{pH}$ da $\mathrm{AU}$, os valores de $\mathrm{SpO}_{2}$ fetal foram estatisticamente diferentes entre os grupos estudados. Pesquisas com maior número de casos devem ser realizadas com vistas à relação existente entre $\mathrm{SpO}_{2}$ fetal e o ponto de corte nos valores de $\mathrm{pH}$ da $\mathrm{AU}^{2}$, para o estabelecimento da preditividade de fetos acidóticos ao nascimento.

\section{SUMMARY}

Purpose: to study fetal oxygen saturation $\left(\mathrm{SpO}_{2}\right)$ levels during labor by continuous pulse oximetry tecnique, and its relation to umbilical artery (UA) $\mathrm{pH}$.

Patients and Methods: fetal $\mathrm{SpO}$ levels were measured during labor by the pulse oximetry technique in 50 subjects. Average values of $\mathrm{SpO}_{2}$ were compared between the first and second stage of labor, with the first stage further subdivided into phases, according to cervical dilatation of $(\leq 4 \mathrm{~cm}, 5-7 \mathrm{~cm}$ and $8-9 \mathrm{~cm}) . \mathrm{SpO}_{2}$ values were studied in relation to umbilical 
artery $\mathrm{pH}$ at birth $(\geq 7.20$ and $<7.20) . \mathrm{SpO}_{2} \geq 30.0 \%$ was considered normal.

Results: fetal $\mathrm{SpO}_{2}$ averages during the first stage were 53.0 $\pm 7.3 \%$ and $44.2 \pm 6.8 \%$ (UA $p H \geq 7.20$ and $<7.20$, respectively; $p<0.01)$. When the first stage was subdivided, the fetal $\mathrm{SpO}$ averages (UA $\mathrm{pH} \geq 7.20$ ) were $55.1 \pm 5.1 \%(\leq 4 \mathrm{~cm}), 52.3 \pm$ $4.6 \%(5-7 \mathrm{~cm})$ and $51.5 \pm 7.2 \%(8-9 \mathrm{~cm}) ;$ for $U A \mathrm{pH}<7.20$, the fetal $\mathrm{SpO}$ averages were $46.3 \pm 5.1 \%(\leq 4 \mathrm{~cm}), 43.6 \pm$ $6.7 \%(5-7 \mathrm{~cm})$ and $42.8 \pm 5.8 \%(8-9 \mathrm{~cm})$. Considering the UA $p H$, these differences were statistically significant $(p<0.01)$. Conclusion: a significant decrease of oxygen saturation values was observed during labor when fetal pulse oximetry was used.

KEY WORDS: Fetal pulse oximetry. Fetal distress. Fetal monitoring.

\section{Referências}

1. Aarnoudse JG, Huisjes HJ, Gordon H, Oeseburg B, Zijlstra WG. Fetal subcutaneous scalp $\mathrm{PO}_{2}$ and abnormal heart rate during labor. Am J Obstet Gynecol 1985; 153:565-6.

2. Banta HD, Thacker SB. Costs and benefits to electronic fetal monitoring: a review of the literature. National Center for Health Services Research, 1979. Report : DHEW-PHS-79-3245.

3. Butterwegge M. Fetal pulse oximetry and nonreassuring heart rate. Eur J Obstet Gynecol Reprod Biol 1997;72 Suppl. 1:S63-6.

4. Clark SL, Gimovsky ML, Miller FC. The scalp stimulation test: a clinical alternative to fetal scalp blood sampling. Am J Obstet Gynecol 1984;148:274-7.

5. Clark SL, Paul RH. Intrapartum fetal surveillance: the role of fetal scalp blood sampling. Am J Obstet Gynecol 1985;153:717-20.

6. Dildy GA, Clark SL, Garite TJ, Porter TF, Swedlow DB, Varner MW. Current status of the multicenter randomized clinical trial on fetal oxygen saturation monitoring in the United States. Eur J Obstet Gynecol Reprod Biol 1997;72 (Suppl 1): S43-50.

7. Dildy GA, Clark SL, Loucks CA. Preliminary experience with intrapartum fetal pulse oximetry in humans. Obstet Gynecol 1993;81:630-5.

8. Dildy GA, van den Berg PP, Katz M, Clark SL, Jongsma HW, Nijhuis JG, Loucks CA. Intrapartum fetal oximetry: fetal oxygen saturation trends during labor and relation to delivery outcome. Am J Obstet Gynecol 1994;171:679-84.

9. Electronic fetal heart rate monitoring: research guidelines for interpretation. The National Institute of Child Health and Human Development Research Planning Workshop. J Obstet Gynecol Neonatal Nurs 1997; 26:635-40.
10. Goffinet F, Langer B, Carbonne B, Berkane N, Tardif $D$, Le Goueff $F$, et al. Multicenter study on the clinical value of fetal pulse oximetry. I Methodologic evaluation. The French Study Group on Fetal Pulse Oximetry. Am J Obstet Gynecol 1997;177:1238-46.

11. Johnson J. Development and potential of fetal pulse oximetry. Contemp Rev Obstet Gynaecol 1991;3:193-200.

12. Johnson N, Johnson VA, Fisher J, Jobbings B, Bannister J, Lilford RJ. Fetal monitoring with pulse oximetry. Br J Obstet Gynaecol 1991;98:3641.

13. Kühnert M, Seelbach-Göebel B, Butterwegge M. Predictive a greement between the fetal arterial oxygen saturation and fetal scalp $\mathrm{pH}$ : results of the German multicenter study. Am J Obstet Gynecol 1998;178:330-5.

14. Langer B, Carbonne B, Goffinet F, Le Gouëff F, Berkane N, Laville M. Fetal pulse oximetry and fetal heart rate monitoring during stage II of labour. Eur J Obstet Gynecol Reprod Biol 1997;72 Suppl. 1: S57-61.

15. Maesel A, Martensson L, Gudmundsson S, Marsal K. Fetal pulse oximetry. A methological study. Acta Obstet Gynecol Scand 1996;75:144-8.

16. Nijland R, Jongsma HW, Nijhuis JG, van den Berg $\mathrm{PP}$, Oeseburg B. Arterial oxygen saturation in relation to metabolic acidosis in fetal lambs. Am J Obstet Gynecol 1995;172:810-9.

17. Oeseburg B, Ringnalda BE, Crevels J, Jongsma HW, Mannheimer P, Menssen J, et al. Fetal oxygenation in chronic maternal hypoxia: what's critical? Adv Exp Med Biol 1992;317:499-502.

18. Richardson BS, Carmichael L, Homan J, Patrick JE. Electrocortical activity, electroocular activity, and breathing movements in fetal sheep with prolonged and graded hypoxemia. Am J Obstet Gynecol 1992;167:553-8.

19. Seelbach-Gobel B, Butterwegge M, Kuhnert M, Heupel M. Fetal reflectance pulse oximetry. Experiences-prognostic significance and consequences-goals. Z Geburtshilfe Perinatol 1994;198:67-71.

20. Small ML, Beall M, Platt LD, Dirks D, Hochberg H. Continuous tissue $\mathrm{pH}$ monitoring in the term fetus. Am J Obstet Gynecol 1989;161:323-9.

21. Van den Berg PP, Dildy GA, Luttkus A, Mason GC, Harvey CJ, Nijhuis JG et al. The efficacy of intrapartum fetal surveillance when fetal pulse oximetry is added to cardiotocography. Eur $\mathrm{J}$ Obstet Gynecol Reprod Biol 1997;72 Suppl. 1:S67-71.

22. Young BK. Continuous fetal tissue $\mathrm{pH}$ monitoring in labor. J Perinat Med 1981;9:189-94. 